\title{
Stress Distribution of Bolted Joints With Different Lay-Up Types
}

\author{
H. Ahmad \\ Faculty of Civil and Environmental Engineering, Universiti Tun Hussein Onn Malaysia, 86400 Parit Raja, Batu Pahat, Johor, Malaysia
}

\begin{abstract}
A parametric study of the stress distribution in the composite plates is undertaken to show the effect of different lay-ups. Full 3-D elastic properties are required in the modelling. These properties are calculated using equations taken from the literature and derivation from simple Classical Laminate Plate Theory (CLPT). In previous experimental work, it was shown that tensile failure involved the development of a damage zone at the edge of the hole. In a double-lap joint, it is assumed that uniform stresses are exhibited throughout the plate thickness. Different lay-ups system give different tangential stress distributions and insignificant variation in the radial stress distribution along the hole boundary.
\end{abstract}

\section{Introduction}

In early finite element work, most of the researchers were working with 2-D finite element models and plane stress state following CLPT theory. The simplified 2D models ignore the effect from the bolt load. Crews, Hong and Raju [1] completed a parametric study on stress distributions around the hole boundary for a variation of $W / d$ values to include bolt properties and contact between bolt and the laminate. Stress distributions are strongly dependent on the anisotropy for both magnitude and location of peak hoop stress on the hole boundary. Stress concentrations for the tested lay-up in the range $2 \leq W / d \leq 10$. The $0^{\circ}$ lay-up gave largest stress concentration (about 4.5), followed by cross-ply lay-up (about 3.75) and the lowest stress concentration is with the quasi-isotropic lay-up (about 1.7). This is consistent with the open-hole problem. This work is later extended to include the effects of friction, pin elasticity, clearance and laminate properties in FEA work by Eriksson et. al [2] and Hyer and Klang [3]. These parameters change the location and value of ultimate tangential stress. Rowlands et. al. [4] compared strain obtained from finite element model to experimental strains using strain gauges on the bearing plane and found that increased friction was able to redistribute the load and correspondingly the position of the main loadcarrying fibres away from the bearing plane towards the net-tension plane.

Lay-up stacking also affects both the bearing strength and failure mode in pin joints as reported by Quinn and Matthews [5] and in clamped bolted joints reported later by Park [6] and found that placing $90^{\circ}$ layers on the surface inhibits delamination bearing strength in the region $\theta=0^{\circ}$ but does not affect the ultimate bearing strength. Stockdale and Matthews [7] reported a $40 \%$ increase in pin loaded bearing strength for a finger-tight case and increased as much as $100 \%$ at a maximum clamping load $(14.7 \mathrm{kN})$ compared to a pin joint in GFRP system. Eriksson [8] found that normalised strength of clamped to pin joint in CFRP system rose by up to 2.4 times. This is expected as lower stiffness in GFRP gives instability effects which reduced its strength performance. Smith [9] found that increasing bolt load changed the initial failure mechanism from local bearing (splitting and interlaminar cracking) to local shear and compression damage under the bolt head resulting in increased bearing stress. Remote bearing failure (more significant in low torque conditions) was shown to be a mixture of compressive damage and delamination failures.

Kontolatis [10] used a similar GFRP woven fabric system as used by Belmonte et. al. [11] in doublelap bolted joints with a clamping torque of $5 \mathrm{~N}$ m using protruding bolt. Kontolatis [10] found that net-tension failures occurred with $W / d \leq 4$. These initiated from the stress concentration at the hole edge perpendicular to the loading axis. Mixed mode of bearing and net-tension failures occurred with $W / d=5$ and sufficiently large $e / d$ $=4$. Kontolatis [10] also conducted interrupted tests to study the effect of net-tension damage in more detail to observe the damage that triggers a catastrophic failure.

An interrupted test in one specimen, from a geometry that finally failed in net-tension, showed that damage in the joint initiated with matrix cracking near the hole edge perpendicular to the loading direction to the specimen sides and at maximum load forms a damage zone of approximately 2-3 mm length. Kontolatis [10] expressed that the damage zone consists of local failures due to discontinuities, including $0^{\circ}$ fibre tow fractures and shear matrix cracking. There were also limited amounts of splitting and delamination formed on both sides of the hole and exhibit a fairly constant width. Exceeding the critical load, the damage zone eventually

\footnotetext{
a Corresponding author: hilton@uthm.edu.my
} 
caused catastrophic failures. Kontolatis [10] also conducted optical microscopy in an attempt to highlight fibre tow fracture in the top and bottom reinforcement layers. The damage zone is localised along the path defined by the crack and the surrounding area seems to be unaffected. The limited amount of splitting may be associated with lateral constraint in torqued conditions in which even the tows that have fractured are retained in place.

In a cross-ply woven fabric laminate, it can be deduced that similar damage growth mechanisms occurred as given in quasi-isotropic lay-ups but due to different weave architectures there is some differences. First, the damage length in the cross-ply lay-up might be less than quasi-isotropic laminates as a result of a high proportion of tows in $0^{\circ}$ direction compared to quasiisotropic laminates with identical thickness. This behaviour also found by Manger [12] in the open-hole problem resulting in reduced critical damage zone length. Secondly, the damage zone tends to initiate at $45^{\circ}$ to the loading axis and then changes to $90^{\circ}$, resulting in shearout failures. As found by Collings [13], sufficient e/d must be provided in cross-ply lay-ups to eliminates the shear-out failures.

Net-tension, shear-out and bearing failures are three main types of failures that do occur in bolted joints. The specific mode of failure is dependent on the stresses developed along radial, tangential and shear plane, respectively. The present work has eliminated shear-out failure by providing sufficient end-distance in all joint systems, so reducing the current study to net-tension and bearing failure modes. Radial stresses are associated with bearing (compressive) failures of loaded hole behind bolt shank and tangential stresses are associated with nettension failures. Current work compares radial stress and tangential stress exhibited in woven fabric CFRP doublelap bolted joints between cross-ply and quasi-isotropic lay-ups. The understanding of stress distribution in composite material lay-ups with bolted joint configurations is essential prior to strength prediction works using finite element analysis.

\section{Experimental Observations}

Net-tension failure occurred within $2<W / d<4$, whereas $W / d=5$ showed bearing mode. Compared with non-woven coupons the transition to bearing failure occurs at a higher $W / d$. It is suggested that this may be due to the lower tensile strength of the woven systems. For coupons tested in the finger-tight condition, the critical $W / d$ is reduced to 4 and local bearing failure prior to ultimate failure is apparent in all samples. The hole elongation is obvious for the coupon with $W / d=5$. Although difficult to see on bare eyes, there was also evidence of local bearing damage in the smaller width coupon. A similar observation with regard to critical $W / d$ is given by Cooper and Turvey [14]. Cooper and Turvey [14] tested pultruded fibre reinforced plastic composite in which critical $W / d$ increased from 4 in pin joint to $W / d=$ 6 with clamping torque $3 \mathrm{~N} \mathrm{~m}$ and $W / d=10$ with clamping torque $30 \mathrm{~N} \mathrm{~m}$.
For the two cross-ply woven materials (PX and 5X) net-tension failure was observed for all the joints tested, except for the unclamped, larger hole size with $W / d=3$. In non-woven materials bearing failure might be expected with $W / d=3$. Again, it is the lower strength of the woven material that is believed to be associated with the greater occurrence of net-tension failures. Two types of bearing failures are observed prior to ultimate failures. The first type showed local bearing initially but ultimate failures are given as net-tension mode (failures given in nettension showed ultimate failures from hole edge and failed catastrophically). This type of bearing failure is more pronounced in thin coupons. Second type showed pure bearing failures which exhibited compression behind the bolt up to certain length (no net-tension failures are observed).

\section{Finite Element Modelling}

\subsection{Determination of 3-D elastic properties}

Determination of 3-D properties is considered first. This is followed by description of the modelling stages which include the use of "smeared-out" properties, the development of the FE models and the associated boundary conditions and the implementation of clampup.

In-plane and out-of-plane elastic properties of the CFRP and GFRP woven fabric composites are required in 3-D modelling. In-plane elastic properties were determined experimentally and are reproduced from Belmonte's work [11] in Table 1. Out-of-plane elastic properties have been estimated following a simple approach. It seems likely that net-tension failure is governed mainly by the in-plane properties. The out-of-plane elastic constants required are $E_{z}, v_{z x}, v_{z y}, G_{z x}$ and $G_{z y}$. For the cross-ply layups these properties are estimated from idealisations based on simple CLPT principles and are presented in Appendix A1. This leads to averaged values over the thickness of the laminate. Out-of-plane properties for the quasi-isotropic lay-ups are taken from Akkerman [15] and given in Appendix A2. The resulting in-plane and out-of-plane elastic properties for all lay-ups are listed in Table 1.

Table 1. Elastic properties of CFRP lay-ups investigated in current study incorporating out-of-plane properties

\begin{tabular}{ccccccc}
\hline $\begin{array}{l}\text { Mate- } \\
\text { rial }\end{array}$ & $\begin{array}{c}\boldsymbol{E}_{\boldsymbol{x}} / \boldsymbol{E}_{\boldsymbol{y}} \\
(\boldsymbol{G P a})\end{array}$ & $\begin{array}{c}\boldsymbol{E}_{z} \\
(\boldsymbol{G P a})\end{array}$ & $\boldsymbol{v}_{\boldsymbol{x y}}$ & $\boldsymbol{v}_{\boldsymbol{y} z} / \boldsymbol{v}_{\boldsymbol{z} \boldsymbol{x}}$ & $\begin{array}{c}\boldsymbol{G}_{\boldsymbol{x} \boldsymbol{y}} \\
(\boldsymbol{G P a})\end{array}$ & $\begin{array}{c}\boldsymbol{G}_{\boldsymbol{y} z} / \boldsymbol{G}_{\boldsymbol{z} \boldsymbol{x}} \\
(\boldsymbol{G P a} \boldsymbol{)}\end{array}$ \\
\hline $\mathrm{PX} 4$ & 51.40 & 12 & 0.09 & 0.10 & 4.42 & 3.90 \\
\hline $\mathrm{PQ} 4$ & 37.19 & 12 & 0.35 & 0.30 & 13.75 & 3.98 \\
\hline
\end{tabular}

In woven composite modelling, the behaviour at the sublamina level is far from fully understood due to the effects of complex fibre architecture. Although much work in predicting mechanical properties of woven fabric systems has been implemented using a unit cell approach, this cannot readily be applied to local failure at a stress raiser. A simple approach that takes into account the 
orthotropic elastic properties of composite materials is by smearing (averaging) to provide "equivalent homogeneous properties". Using smeared properties for a woven fabric system is inherently a better approximation than for non-woven systems because two separate plies in a pre-preg based laminate are replaced by a single layer in a woven fabric composite.

\subsection{Modeling Techniques and Approaches}

The elastic properties used in the current model are based on smeared-out properties and include the out-ofplane elastic properties given in Table 1. Cross-ply woven lay-up is arguably better represented as smearedout than a quasi-isotropic woven system because of similar repetitive sequence in adjacent layers. The other components are made from isotropic stainless steel which has a modulus elasticity of $210 \mathrm{~N} / \mathrm{mm}^{2}$ and Poisson's ratio of 0.3 . Six components are assembled in each model of the single-lap joint. Sufficient mesh refinement is used to ensure the strength predictions are mesh independent. Mesh is refined in the vicinity of the hole edge in the composite (and steel) plates and the volume under the washers, as ultimate failure occurred within these regions. The boundary conditions are assigned so that left end is held fixed and displacement is applied to the right end.

Two load steps are implemented which apply the clamping load (Step 1) and the far-field tensile load (Step 2). Each model is assigned a clamping load (finger-tight or $\mathrm{T}=5 \mathrm{~N} \mathrm{~m}$ ). Each contact surface pair is assigned with master-slave interaction which includes an appropriate friction coefficient. This is an important step as load transfer will affect the stress distributions and therefore, strength predictions.

\section{Results and Discussion}

The stress distribution around the circumference of the bolt hole of cross-ply laminates is somewhat different from quasi-isotropic materials due to the lower ratio of shear modulus-to-longitudinal modulus. In open hole problem, quasi-isotropic lay-up showed a lower stress concentration than cross-ply lay-up. However, in a bolted joint problem, the stress concentration is reduced at low bearing stress due to the effect of friction as a result of lateral load in the vicinity of the hole edge.

To investigate lay-up effects in bolted joints further, two different lay-ups of similar thickness (PX4 and PQ4) are compared giving the results shown in Figure 1. 3-D models are able to model the full friction transfer condition correctly giving zero stress in radial and tangential direction around the hole perimeter for low levels of bearing stress. As can be observed from Figure 1 (a) and (b), increased clamping force will reduce both the radial stress and tangential stress. Both lay-up types does not showed much difference in radial stress distribution around hole boundary where maximum radial stress occurs at $45^{\circ}$ from the bearing as given in Figure 1 (a).
The different lay-ups give different tangential stress distributions along the hole boundary as shown in Figure 1(b). Quasi-isotropic laminate has $\pm 45^{\circ}$ plies and exhibits a single peak in the tangential stress, which increased from bearing plane to peak at about $85^{\circ}$ and reduces subsequently, showing the maximum tensile stress at the end of contact regions. Cross-ply lay-ups have no $\pm 45^{\circ}$ plies and showed a minimum stress at angle of $45^{\circ}$ from bearing plane. Additionally, two peaks are produced at $0^{\circ}$ and $90^{\circ}$ associated with $(0 / 90)_{\text {ns }}$ lay-up where the second tensile stress peak was larger than the first peak and occurred at a location where the non-contact point started. These trends are a consequence of the local changes in lay-up geometry around the hole boundary in a polar co-ordinate system.

Figure 1 (c) shows the tangential stress along net-section; for a fixed bearing stress the tangential stress reduced with bolt load. With fixed bearing stress, lower bolting forces increase the tangential stress concentration. Larger tensile stresses at net-tension plane in cross-ply were due to having proportionately more $0^{\circ}$ plies. Both lay-ups showed insignificant variation in the radial stress distribution. Similar trends were obtained by Crews et. al. [1] who studied the stresses using a 2-D model for a bolted joint with six different (non-woven) laminate layups and found that there was strong anisotropy in magnitude and location of peak tangential stress.

\section{Conclusions}

A 3-D FEA has been carried out to determine the stress distribution in bolted double-lap joints, as friction load in bolted joint is transferred through-the-thickness, the 3-D finite element model gave more reliable stress distribution. Different lay-ups give different tangential stress distributions along the hole boundary, cross-ply lay-up demonstrates higher tangential stress than quasiisotropic lay-up counterparts. This is consequence of the local changes in lay-up geometry around the hole boundary in a polar co-ordinate system. On the other hand, both cross-ply and quasi-isotropic lay-ups showed insignificant variation in the radial stress distribution.

\section{Acknowledgements}

The authors would like to be obliged to Universiti Tun Hussein Onn Malaysia and Ministry of Education Malaysia for providing laboratory facilities and financial assistance under project Vot no. R034.

\section{References}

[1] Crews, J.H., Hong, C.S., and Raju, I.S.,. Stress Concentration Factors for Finite Orthotropic Laminates with a Pin-loaded Hole. 1-40, 1981: NASATechnology Paper 1862, 1981.

[2] Eriksson, L.I.,. "Contact Stresses in Bolted Joints of Composite Laminates." Composite Structures, 1986: 6:57-75. 
[3] Hyer, M.W., Klang, E.C. and Cooper, D.E.,. "The Effects of Pin Elasticity, Clearance and Friction on the Stresses in a Pin-loaded Orthotropic Plate." Journal Composite Material, 1987: 21:190-206.

[4] Rowlands, R.E., Rahman, M.U., Wilkinson, T.L., Chiang, Y.I.,. "Single and Multiple-bolted Joints in Orthotropic Materials." Composites, 1982: 13:273279.

[5] Quinn, W.J., Matthews, F.L.,. "The Effect of Stacking Sequence on the Pin-Bearing Strength in Glass Fibre Reinforced Plastic." Journal of Composite Materials, 1977: 11:139-145.

[6] Park, H.J.,. "Effects of Stacking Sequence and Clamping Force on the Bearing Strengths of Mechanically Fastened Joints in Composite Laminates." Composite Structures, 2001: 53:213221.

[7] Stockdale, J.H., Matthews, F.L.,. "The Effect of Clamping Pressure on Bolt Bearing Loads in Glass Fibre Reinforced Plastics." Composites, 1976: 3439.

[8] Eriksson, I.,. "An Analysis Method for Bolted Joints in Primary Composite." AGARD Conference Proceedings 427. Madrid, Spain, 1987. 6.1-6.19.

[9] Smith, P.A.,. Aspects of the Static and Fatigue Behaviour of Composite Laminates, including Bolted Joints. PhD thesis, Cambridge, UK: Cambridge University, 1985.

[10] Kontolatis, A.,. Failure of Composite Bolted Joints Made from Woven fabric GFRP Composite. MSc dissertation, Guildford: University of Surrey, 2000.

[11] Belmonte, H.M.S., Manger, C.I.C., Ogin, S.L., Smith, P.A., Lewin, R.,. "Characterisation and Modeling of the Notched Tensile Fracture of Woven Quasi-isotropic GFRP Laminates." Composites Science and Technology, 2001: 61:585-597.

[12] Manger, C.I.C.,. Failure of Notched Woven GFRP Composites: Damage Analysis and Strength Modelling. PhD Thesis, Guildford: University of Surrey, 1999.

[13] Collings, T.A.,. "The Strength of Bolted Joints in Multi-directional CFRP Laminates." Composites, 1977: 43-55.

[14] Cooper, C., Turvey, G.J.,. "Effects of Joint Geometry and Bolt Torque on the Structural Performance of Single Bolt Tension Joints in Pultruded GRP Sheet Material." Composite Structures, 1995: 32:217-226.

[15] Akkerman, R.,. "On the Properties of Quasiisotropic Laminates." Composites: Part B, 2002: 33:133-140. 


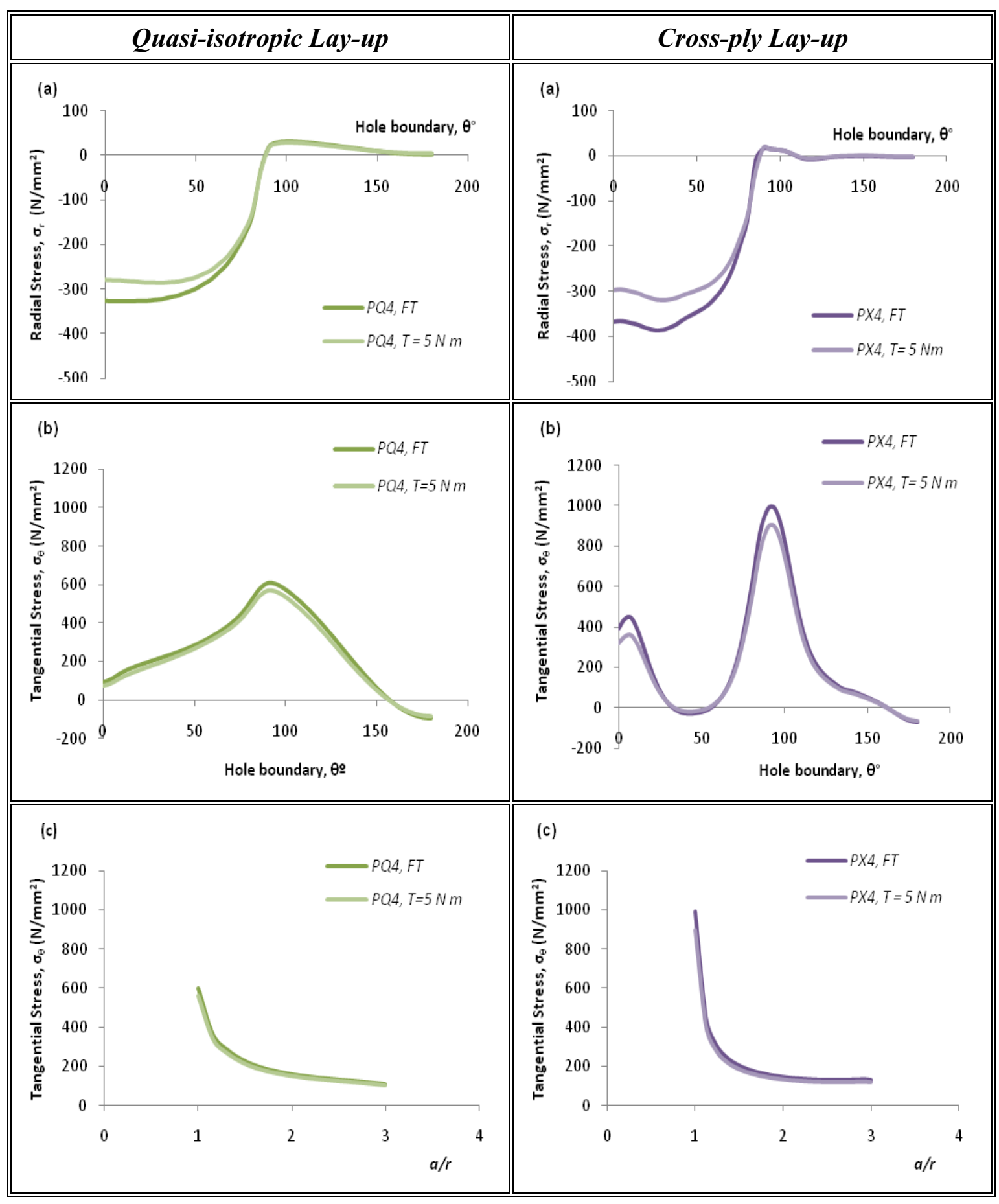

Figure 1. Comparison of FEA stress distributions near loaded hole in quasi-isotropic (PQ4) and cross-ply (PX4) lay-ups with $d=10 \mathrm{~mm}$ in the finger-tight and clamped conditions at an applied bearing stress of $400 \mathrm{~N} / \mathrm{mm}^{2}$ (a) Radial stress around hole boundary (b) Tangential stress around hole boundary (c) Tangential stress with normalised distance from the hole edge.

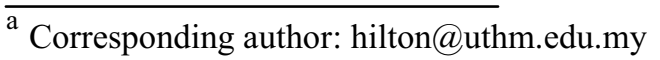




\section{Appendix A: Determination of out-of-plane elastic constants}

\section{A1: Cross-ply laminates}

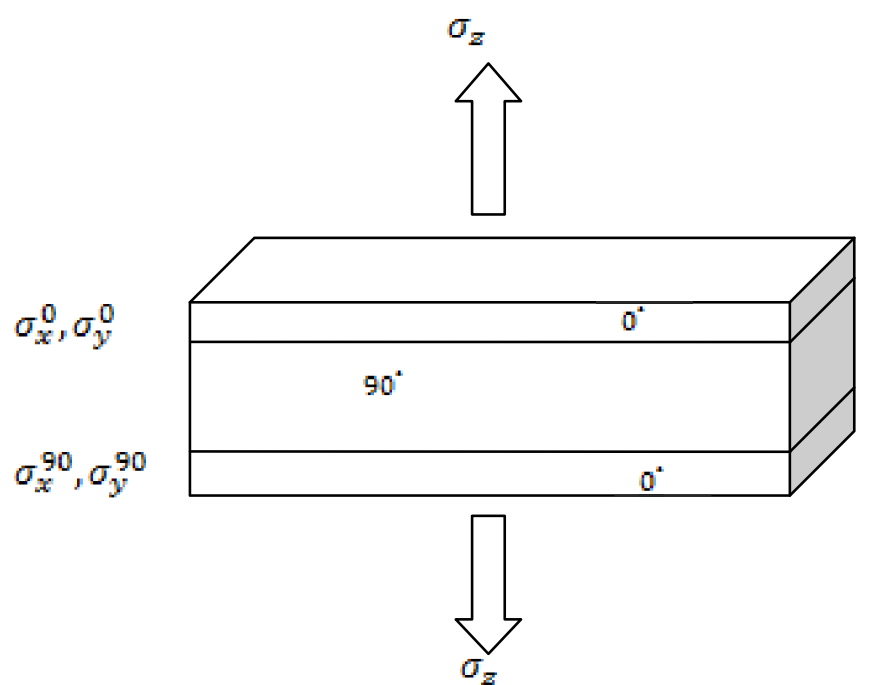

For equal thickness of

0 's and 90's

$\sigma_{x}^{0}+\sigma_{x}^{90}=0$

$\sigma_{y}^{0}+\sigma_{y}^{90}=0$

Now $\sigma_{z}$ is constant then the thickness

$\varepsilon_{z}^{0}=\frac{\sigma_{z}}{E_{3}}-\frac{v_{13} \sigma_{x}^{0}}{E_{1}}-\frac{v_{23} \sigma_{y}^{0}}{E_{2}}$
$\varepsilon_{z}^{90}=\frac{\sigma_{z}}{E_{3}}-\frac{v_{23} \sigma_{x}^{90}}{E_{2}}-\frac{v_{13} \sigma_{y}^{90}}{E_{1}}$

Effective stresses in z-direction,

$$
\begin{aligned}
& \overline{\varepsilon_{z}}=\frac{\varepsilon_{z}^{0}+\varepsilon_{z}^{90}}{2} \\
& \overline{\varepsilon_{z}}=\frac{\sigma_{z}}{E_{z}}=\frac{\sigma_{z}}{E_{3}}-\frac{1}{2}\left[\frac{v_{13}}{E_{1}}\left(\sigma_{x}^{0}+\sigma_{y}^{90}\right)+\frac{v_{23}}{E_{2}}\left(\sigma_{x}^{90}+\sigma_{y}^{0}\right)\right]
\end{aligned}
$$

Also have strain compatibility in the $\mathrm{x}-$ and $\mathrm{y}$ - direction,

$$
\begin{aligned}
& \varepsilon_{x}^{0}=\frac{\sigma_{x}^{0}}{E_{1}}-\frac{v_{21} \sigma_{y}^{0}}{E_{2}}-\frac{v_{31} \sigma_{z}}{E_{3}} \\
& \varepsilon_{x}^{90}=\frac{\sigma_{x}^{90}}{E_{2}}-\frac{v_{12} \sigma_{y}^{90}}{E_{1}}-\frac{v_{23} \sigma_{z}}{E_{3}} \\
& \varepsilon_{y}^{0}=\frac{\sigma_{y}^{0}}{E_{2}}-\frac{v_{12} \sigma_{x}^{0}}{E_{1}}-\frac{v_{23} \sigma_{z}}{E_{3}} \\
& \varepsilon_{y}^{90}=\frac{\sigma_{y}^{90}}{E_{1}}-\frac{v_{21} \sigma_{x}^{90}}{E_{2}}-\frac{v_{31} \sigma_{z}}{E_{3}}
\end{aligned}
$$

As composite shows similar strain in each layer, $\varepsilon_{x}^{0}=\varepsilon_{x}^{90}$,

$$
\frac{\sigma_{x}^{0}}{E_{1}}-\frac{v_{21} \sigma_{y}^{0}}{E_{2}}-\frac{v_{31} \sigma_{z}}{E_{3}}=\frac{\sigma_{x}^{90}}{E_{2}}-\frac{v_{12} \sigma_{y}^{90}}{E_{1}}-\frac{v_{23} \sigma_{z}}{E_{3}}
$$


$\frac{\sigma_{y}^{0}}{E_{2}}-\frac{v_{12} \sigma_{x}^{0}}{E_{1}}-\frac{v_{23} \sigma_{z}}{E_{3}}=\frac{\sigma_{y}^{90}}{E_{1}}-\frac{v_{21} \sigma_{x}^{90}}{E_{2}}-\frac{v_{31} \sigma_{z}}{E_{3}}$

From equilibrium as given by $\mathrm{A} 1$ and $\mathrm{A} 2$,

$$
\begin{aligned}
& \sigma_{x}^{0}=-\sigma_{x}^{90} \\
& \sigma_{y}^{0}=-\sigma_{y}^{90}
\end{aligned}
$$

If we substitute (A3) and (A4) into (A1) and (A2), it should be possible to solve for $\sigma_{x}^{90}, \sigma_{y}^{90}$ in terms of $\sigma_{z}$,

$$
\begin{aligned}
& \frac{-\sigma_{x}^{90}}{E_{1}}+\frac{v_{21} \sigma_{y}^{90}}{E_{2}}+\frac{\sigma_{z}}{E_{3}}\left(v_{23}-v_{31}\right)=\frac{\sigma_{x}^{90}}{E_{2}}-\frac{v_{12} \sigma_{y}^{90}}{E_{1}} \\
& \sigma_{x}^{90}\left(\frac{1}{E_{1}}+\frac{1}{E_{2}}\right)-\sigma_{y}^{90}\left(\frac{v_{12}}{E_{1}}+\frac{v_{21}}{E_{2}}\right)=\frac{\sigma_{z}}{E_{3}}\left(v_{23}-v_{31}\right) \\
& \frac{-\sigma_{y}^{90}}{E_{2}}+\frac{v_{12} \sigma_{x}^{90}}{E_{1}}-\frac{\sigma_{z}}{E_{3}}\left(v_{23}-v_{31}\right)=\frac{\sigma_{y}^{90}}{E_{1}}-\frac{v_{21} \sigma_{x}^{90}}{E_{2}} \\
& \sigma_{x}^{90}\left(\frac{v_{12}}{E_{1}}+\frac{v_{21}}{E_{2}}\right)-\sigma_{y}^{90}\left(\frac{1}{E_{1}}+\frac{1}{E_{2}}\right)=\frac{\sigma_{z}}{E_{3}}\left(v_{23}-v_{31}\right)
\end{aligned}
$$

By inspection,

$$
\begin{gathered}
\sigma_{x}^{90}=\sigma_{y}^{0} \\
\sigma_{y}^{90}=\sigma_{x}^{0} \\
\overline{\varepsilon_{z}}=\frac{\sigma_{z}}{E_{z}}=\frac{\sigma_{z}}{E_{3}}-\frac{v_{13}}{E_{1}} \sigma_{x}^{0}-\frac{v_{23}}{E_{2}} \sigma_{x}^{90} \\
=\frac{\sigma_{z}}{E_{3}}-\frac{v_{13}}{E_{1}} \sigma_{x}^{0}-\frac{v_{23}}{E_{2}}\left(-\sigma_{x}^{0}\right) \\
=\frac{\sigma_{z}}{E_{3}}+\left(\frac{v_{23}}{E_{2}}-\frac{v_{13}}{E_{1}}\right) \sigma_{x}^{0}
\end{gathered}
$$

Determination of $E_{z}$

From (A5),

$$
\begin{aligned}
& \sigma_{x}^{90} \frac{E_{1}+E_{2}}{E_{1} E_{2}}-\sigma_{y}^{90} \frac{2 v_{12}}{E_{1}}=\frac{\sigma_{z}}{E_{3}}\left(v_{23}-v_{31}\right) \\
& -\sigma_{x}^{0}\left(\frac{E_{1}+E_{2}}{E_{1} E_{2}}\right)-\sigma_{x}^{0} \frac{2 v_{12}}{E_{1}}=\frac{\sigma_{z}}{E_{3}}\left(v_{23}-v_{31}\right) \\
& \sigma_{x}^{0}\left[\frac{2 v_{12}}{E_{1}}+\left(\frac{E_{1}+E_{2}}{E_{1} E_{2}}\right)\right]=-\frac{\sigma_{z}}{E_{3}}\left(v_{23}-v_{31}\right) \\
& \sigma_{x}^{0}=-\frac{\left(v_{23}-v_{31}\right)}{E_{3}\left[\frac{2 v_{12}}{E_{1}}+\left(\frac{E_{1}+E_{2}}{E_{1} E_{2}}\right)\right]} \sigma_{z}
\end{aligned}
$$




$$
\begin{aligned}
& \frac{\sigma_{z}}{E_{z}}=\frac{\sigma_{z}}{E_{3}}-\frac{\left(v_{23}-v_{31}\right)\left(\frac{v_{23}}{E_{2}}-\frac{v_{13}}{E_{1}}\right)}{E_{3}\left[\frac{2 v_{12}}{E_{1}}+\left(\frac{E_{1}+E_{2}}{E_{1} E_{2}}\right)\right]} \sigma_{z} \\
& \frac{\sigma_{z}}{E_{z}}=\sigma_{z} \frac{1}{E_{3}}\left[1-\frac{\left(v_{23}-v_{31}\right)\left(\frac{v_{23}}{E_{2}}-\frac{v_{13}}{E_{1}}\right)}{\left[\frac{2 v_{12}}{E_{1}}+\left(\frac{E_{1}+E_{2}}{E_{1} E_{2}}\right)\right]}\right] \\
& E_{z}=\frac{E_{3}}{\left[1-\frac{\left(v_{23}-v_{31}\right)\left(\frac{v_{23}}{E_{2}}-\frac{v_{13}}{E_{1}}\right)}{\left[\frac{2 v_{12}}{E_{1}}+\left(\frac{E_{1}+E_{2}}{E_{1} E_{2}}\right)\right]}\right]}
\end{aligned}
$$

Determination of $v_{z x}$

$v_{z x}=\frac{\varepsilon_{x}}{\varepsilon_{z}}=\frac{\text { Lateral strain }}{\text { Axis strain }}$

$=-\frac{\frac{\sigma_{x}^{0}}{E_{1}}-\frac{v_{21} \sigma_{y}^{0}}{E_{2}}-\frac{v_{31} \sigma_{z}}{E_{3}}}{\frac{\sigma_{z}}{E_{3}}-\frac{1}{2}\left[\frac{v_{13}}{E_{1}}\left(\sigma_{x}^{0}+\sigma_{y}^{90}\right)+\frac{v_{23}}{E_{1}}\left(\sigma_{x}^{90}+\sigma_{y}^{0}\right)\right]}$

$=-\frac{\frac{\sigma_{x}^{0}}{E_{1}}+\frac{v_{21} \sigma_{x}^{0}}{E_{2}}-\frac{v_{31} \sigma_{z}}{E_{3}}}{\frac{\sigma_{z}}{E_{3}}-\frac{v_{13} \sigma_{x}^{0}}{E_{1}}+\frac{v_{23} \sigma_{x}^{0}}{E_{2}}}$

From (A7),

$$
\begin{aligned}
& \sigma_{x}^{0}=-\frac{\left(v_{23}-v_{31}\right)}{E_{3}\left[\frac{2 v_{12}}{E_{1}}+\left(\frac{E_{1}+E_{2}}{E_{1} E_{2}}\right)\right]} \sigma_{z}=\frac{-\left(v_{23}-v_{31}\right) \sigma_{z} E_{1} E_{2}}{E_{3}\left(2 v_{12} E_{2}+E_{1}+E_{2}\right)} \\
& =-\left[\frac{\frac{-\left(v_{23}-v_{31}\right) \sigma_{z} E_{2}^{2} E_{1}}{2 v_{12} E_{2}+E_{1}+E_{2}}-\frac{v_{21}\left(v_{23}-v_{31}\right) \sigma_{z} E_{1}^{2} E_{2}}{2 v_{12} E_{2}+E_{1}+E_{2}}-v_{31} \sigma_{z} E_{1} E_{2}}{\sigma_{z} E_{1} E_{2}+\frac{\left(v_{23}-v_{31}\right) v_{13} \sigma_{z} E_{2}^{2} E_{1}}{2 v_{12} E_{2}+E_{1}+E_{2}}-\frac{\left(v_{23}-v_{31}\right) v_{23} \sigma_{z} E_{1}^{2} E_{2}}{2 v_{12} E_{2}+E_{1}+E_{2}}}\right]
\end{aligned}
$$

Diminished the dominator and $\sigma_{z}$

$$
\begin{aligned}
& v_{z x}=-\left[\frac{-\left(v_{23}-v_{31}\right) E_{2}^{2} E_{1}-v_{21}\left(v_{23}-v_{31}\right) E_{1}^{2} E_{2}-v_{31} E_{1} E_{2}\left(2 v_{12} E_{2}+E_{1}+E_{2}\right)}{\left(2 v_{12} E_{2}+E_{1}+E_{2}\right) E_{1} E_{2}+\left(v_{23}-v_{31}\right) v_{13} E_{2}^{2} E_{1}-\left(v_{23}-v_{31}\right) v_{23} E_{1}^{2} E_{2}}\right] \\
& v_{z x}=-\left[\frac{-\left(v_{23}-v_{31}\right) E_{2}-v_{21}\left(v_{23}-v_{31}\right) E_{1}-v_{31}\left(2 v_{12} E_{2}+E_{1}+E_{2}\right)}{\left(2 v_{12} E_{2}+E_{1}+E_{2}\right)+\left(v_{23}-v_{31}\right) v_{13} E_{2}-\left(v_{23}-v_{31}\right) v_{23} E_{1}}\right]
\end{aligned}
$$




\section{A2: Quasi-Isotropic laminates}

*For quasi-isotropic, the exact formulation are given in Akkermann (2002)

$$
\begin{array}{lc}
E_{x}=E_{y} & \begin{array}{c}
2\left(1+v_{x y}\right) G_{x y} \\
E_{z}
\end{array} \\
\frac{G_{x z}}{\left(1-v_{23}^{2}\right) \frac{E_{1}}{E_{2}}+\left(1+2 v_{12}+2 v_{12} v_{23}\right)-v_{12}^{2} \frac{E_{2}}{E_{1}}} \\
v_{x z}=v_{y z} & 2\left(\frac{G_{12} G_{28}}{G_{12}+G_{23}}\right) \\
& \frac{E_{x}}{E_{1}} \cdot \frac{\left(v_{12}+v_{23}+v_{12} v_{13}\right)+v_{12}^{2} \frac{E_{2}}{E_{1}}}{1+\left(1+2 v_{12}\right) \frac{E_{2}}{E_{1}}}
\end{array}
$$

